\title{
Capecitabine plus Irinotecan (XELIRI regimen) compared to 5-FU/LV plus Irinotecan (FOLFIRI regimen) as neoadjuvant treatment for patients with unresectable liver-only metastases of metastatic colorectal cancer: a randomised prospective phase II trial
}

\author{
Erik Skof ${ }^{\dagger}$, Martina Rebersek $^{\dagger}$, Zvezdana Hlebanja $^{\dagger}$ and Janja Ocvirk*†
}

Address: Institute of Oncology, Division of Medical Oncology, Zaloska 2, 1000 Ljubljana, Slovenia

Email: Erik Skof - eskof@onko-i.si; Martina Rebersek - mrebersek@onko-i.si; Zvezdana Hlebanja - zhlebanja@onko-i.si; Janja Ocvirk* - jocvirk@onko-i.si

* Corresponding author †Equal contributors

Published: 22 April 2009

BMC Cancer 2009, 9:120 doi:10.1 186/147|-2407-9-120
Received: 6 August 2008

Accepted: 22 April 2009

This article is available from: http://www.biomedcentral.com/I47/-2407/9//20

(c) 2009 Skof et al; licensee BioMed Central Ltd.

This is an Open Access article distributed under the terms of the Creative Commons Attribution License (http://creativecommons.org/licenses/by/2.0), which permits unrestricted use, distribution, and reproduction in any medium, provided the original work is properly cited.

\begin{abstract}
Background: Phase II studies have shown that the combination of capecitabine and irinotecan (the XELIRI regimen) is active in metastatic colorectal cancer (MCRC). There are, however, no data about the use of the XELIRI regimen in the neoadjuvant treatment.

Methods: Patients with unresectable liver-only metastases of MCRC with $\leq 75$ years of age were randomised to either the XELIRI (irinotecan $250 \mathrm{mg} / \mathrm{m}^{2}$ given on day one and capecitabine 1000 $\mathrm{mg} / \mathrm{m}^{2}$ twice daily from day $2-15$, every $2 I$ days) or the FOLFIRI arm (irinotecan $180 \mathrm{mg} / \mathrm{m}^{2}, 5-\mathrm{FU}$ $400 \mathrm{mg} / \mathrm{m}^{2}$, LV $200 \mathrm{mg} / \mathrm{m}^{2}, 5$-FU $2400 \mathrm{mg} / \mathrm{m}^{2}$ (46-h infusion) - all given on day one, every I 4 days). Primary end points were objective response rate (ORR) and rate of radical (R0) surgical resection. Secondary end points were progression-free survival (PFS), overall survival (OS) and safety.
\end{abstract}

Results: Altogether 87 patients were enrolled (4I pts in the XELIRI and 46 pts in the FOLFIRI arm). The median age was 63 years ( 63 years in the XELIRI and 62 years in the FOLFIRI arm) $(p=$ 0.33). ORR was $49 \%$ in the XELIRI and $48 \%$ in the FOLFIRI arm $(p=0.76)$. The rate of radical RO resection was $24 \%$ in both arms of patients. At the end of treatment, $37 \%$ of patients in the XELIRI and $26 \%$ of patients in the FOLFIRI arm were without evidence of the disease (CR+RO resection) $(p=0.56)$. There were no statistical differences in grade 3 or 4 adverse events between both arms: diarrhoea $7 \%$ vs. $6 \%$, neutropenia $5 \%$ vs. $13 \%$, ischemic stroke 0 vs. $2 \%$, acute coronary syndrome $2 \%$ vs. $4 \%$, respectively. At the median follow up of 17 (range I-39) months, the median PFS was I0.3 months in the XELIRI and 9.3 months in the FOLFIRI arm $(p=0.78)$, the median OS was 30.7 months in the XELIRI arm and 16.6 months in the FOLFIRI arm $(p=0.16)$.

Conclusion: The XELIRI regimen showed similar ORR as the FOLFIRI regimen in the neoadjuvant treatment of patients with MCRC. In addition, the XELIRI regimen showed similar PFS and OS with acceptable toxicity compared to the FOLFIRI regimen.

Trial Registration: Current Controlled Trials ISRCTNI9912492 


\section{Background}

The majority of patients with metastatic colorectal cancer (MCRC) have liver metastases. Radical (R0) resection of liver metastases offers the greatest likelihood of cure in the patients with liver-only metastases of MCRC. The five-year survival rates after radical resection are $24-58 \%$ [1-4], whereas the five-year survival rates for unresectable disease with most active systemic chemotherapy regimens are $<5 \%$ [5]. Since the majority of patients with MCRC have unresectable liver metastases, neoadjuvant (or preoperative) chemotherapy is an appropriate treatment choice with intent to reduce the number and/or size of the liver metastases to make the radical (R0) resection of liver metastases possible. This can be achieved in 12-33\% of patients [6-8]. In the neoadjuvant chemotherapy, combinations of fluoropyrimidines (5-FU/leucovorin (LV)) with irinotecan and/or oxaliplatin are usually used. Among the combinations of fluoropyrimidines with irinotecan, the FOLFIRI regimen is preferred, whereas the FOLFOX regimen is preferred among the combinations of fluoropyrimidines with oxaliplatin. Randomised studies have shown that the combination of capecitabine with oxaliplatin (XELOX regimen) in the first-line treatment of patients with MCRC shows similar efficacy and tolerability compared to the combinations of 5-FU/LV with oxaliplatin $[9,10]$. There are, however, only few published data about the combination of capecitabine with irinotecan in the treatment of patients with MCRC. Phase I/II studies have demonstrated that the combination of capecitabine (1000 mg/m2 twice daily for 14 days) with irinotecan ( $250 \mathrm{mg} / \mathrm{m} 2$ i.v. on day one, every 21 days) (the XELIRI regimen) is active [11-14]. The XELIRI regimen showed response rates of $35-54 \%$ and time to progression $8-9$ months. Most common treatment-related grade 3 or grade 4 adverse events reported from a phase II study were neutropenia (25\%), diarrhoea (20\%), vomiting (16\%), dehydration $(10 \%)$, nausea $(6 \%)$, abdominal pain $(6 \%)$, and hand-foot syndrome (6\%) [11].

To our knowledge, there has been no study published with the XELIRI regimen in the neoadjuvant setting of patients with unresectable liver-only metastases of MCRC. The aim of our study was to compare the efficacy, safety and survival of the XELIRI regimen to the standard FOLFIRI regimen in the neoadjuvant setting of patients with unresectable liver-only metastases of MCRC.

Our hypothesis was that there are no statistically significant differences in efficacy, survival and safety of the XELIRI regimen compared to the standard FOLFIRI regimen. However, the XELIRI regimen seems to be more convenient when compared to the FOLFIRI regimen since capecitabine is an oral drug; therefore, there is no need for central venous catheters implantation (risk for bleeding, infection, thrombosis) and no need for hospitalisation.
The treatment is performed in out-patient clinic. The cycles are applied every 21 days compared to every 14 days in the FOLFIRI regimen.

In the year 2004, at the time when our study was initiated, bevacizumab was not yet registered for the treatment of patients with MCRC in Slovenia. However, in 2006, while our study was ongoing, bevacizumab became a standard in the first-line treatment of patients with MCRC. This was the main reason why the study was prematurely closed for the accrual at the end of the year 2006 with only $43 \%$ of initially planned accrual.

\section{Methods}

The study was performed at the Institute of Oncology Ljubljana, Slovenia, after it had been approved by National Medical Ethics Committee. This was a prospective randomised phase II study.

Eligibility criteria were: age 18-75 years, performance status of $0-1$ according to $\mathrm{WHO}$, unresectable liver metastases of colorectal adenocarcinoma - determined by liver surgeon either because of the size, number, or unfavourable location of metastases that did not allow a complete resection of disease leaving at least $25 \%$ of normal liver parenchyma, no prior chemotherapy for metastatic disease, $>6$ months since adjuvant treatment, at least one measurable lesion of $\geq 1 \mathrm{~cm}$ visible on spiral computed tomography (CT), bilirubin $<2 \times$ times the upper limit of normal (ULN), aspartate aminotransferase (AST) $<5 \times$ ULN, alanine aminotransferase (ALT) $<5 \times$ ULN, adequate haematological and renal function, and signed informed consent.

Exclusion criteria were: extra-hepatic disease (either metastases outside liver or loco-regional recurrence), other malignancy within the past 5 years (except limited basal cell or squamous cell carcinoma of the skin or in situ cervical carcinoma), inadequately controlled hypertension (blood pressure $>150 / 100 \mathrm{mmHg}$ on antihypertensive medications), unstable angina pectoris, history of myocardial infarction or stroke within 6 months, clinically significant peripheral vascular disease, inflammatory bowel disease. Patients that fulfilled eligibility criteria were randomly assigned to either the XELIRI or FOLFIRI arm with no prior stratification.

\section{Patient evaluation}

Pre-treatment evaluation included a detailed medical history, physical examination, a complete blood count with differential and platelet count, blood chemistry, serum levels of carcinoembryonic antigen (CEA) and baseline tumour measurements by CT. The pre-treatment evaluation had to be performed within 14 days before the treatment was initiated. The patients were assessed for toxicity 
before each application of cytotoxic drugs. Repeat imaging was required every three months during treatment. Chemotherapy was discontinued prior to the expiry of six months if liver metastases became resectable (patients were referred to liver surgeon) or if there was either progression of the disease or serious adverse event occurred during chemotherapy. After the discontinuation of study treatment, a follow-up examination, including clinical examination, blood samples (liver enzymes, CEA), CT or ultrasound of the abdomen, was performed every 3 months, until the progression of the disease or death.

\section{Chemotherapy}

The XELIRI regimen consisted of irinotecan $250 \mathrm{mg} / \mathrm{m}^{2}$ given on day one and capecitabine $1000 \mathrm{mg} / \mathrm{m}^{2}$ twice daily from day $2-15$, every 21 days. The FOLFIRI regimen consisted of irinotecan $180 \mathrm{mg} / \mathrm{m}^{2}, 5-\mathrm{FU} 400 \mathrm{mg} / \mathrm{m}^{2}$, LV $200 \mathrm{mg} / \mathrm{m}^{2}, 5$-FU $2400 \mathrm{mg} / \mathrm{m}^{2}$ (46-h infusion) - all given on day one, every 14 days. The patients in both arms received premedication with dexamethason $20 \mathrm{mg}$ i.v., granisetron $1 \mathrm{mg}$ i.v. and diazepam $10 \mathrm{mg}$ i.v. on day 1 of each chemotherapy cycle. To initiate a cycle of cytotoxic chemotherapy, an absolute neutrophil count of at least $1500 / \mu \mathrm{l}$, platelets at least $100000 / \mu \mathrm{l}$ and resolution of other toxic effects to at least CTC grade 1 were required. A resolution of toxicity to at least CTC grade 1 was required within 3 weeks of the intended start of a cycle or patients were withdrawn from the study. All patients were advised to use emollients with urea to manage hand-foot syndrome of grade I and II. The maximum planned duration of the treatment was six months in both arms. Postoperative chemotherapy was not planned.

\section{Surgery}

At evaluation, surgical resection of metastases was reconsidered by a team of experts, consisting of a liver surgeon, radiologist and medical oncologist. Liver surgery was attempted when technically feasible and potentially curative in the patients fit for operative procedure. During surgery, a complete exploration of the abdomen, including intraoperative ultrasound, was performed. Different surgical techniques were used for the resection of metastases.

The primary end points were: overall objective response (ORR) and rate of radical surgical resection (R0 resection). The evaluation of response was based on RECIST [15]. The radical surgical resection ( $\mathrm{R} 0$ resection) of liver metastases was defined as tumour-free margin of $>10 \mathrm{~mm}$ at histology specimen and no signs of residual metastases during exploration of the abdomen. In case of complete response (CR), liver surgery was not performed; regular follow-up was performed in these patients.

Secondary end points were: progression-free survival (PFS), overall survival (OS) and safety. PFS was defined as the time between the randomisation and the progression of the disease or death of any cause; the patients who were withdrawn from the study treatment for other reasons were censored at the discontinuation of study therapy. OS was defined as the time between the randomisation and death. Treatment-related death was defined as the death within 30 days after the last cycle of chemotherapy or liver surgery. All toxic effects were graded according to the National Cancer Institute (NCI) - Common Toxicity Criteria (CTC), version 3.0. The histology reports documented any signs of steatohepatitis.

\section{Statistical considerations}

For the statistical power calculation, online DSS Research toolkit was used. For the FOLFIRI arm, a response rate of $40 \%$ and a R0 resection rate of $15 \%$ was expected. The statistical power for initially planned 200 patients, with Alpha Error level of 5\% (a 95\% Confidence Interval) to show $10 \%$ difference between arms, was calculated to be $41 \%$ for the objective response rate, and $55 \%$ for the R0 resection rate. With 87 patients enrolled, the statistical power for the objective response rate was $24 \%$, and for the $\mathrm{R} 0$ resection rate $32 \%$.

Statistical analysis was performed with the program SPSS - Version 1.3. For the comparison of the two arms, twosided Pearson chi-square test and Student's t-test were used. Objective response rate was assessed on intention to treat (ITT) population. Safety analysis was performed on the group of patients who received at least one dose of protocol medication. Overall survival and progressionfree survival were estimated according to Kaplan-Meier method [16]. Survival curves were compared with the logrank test. Statistical difference between the arms was determined as $\mathrm{p}<0.05$.

\section{Results}

In the period of 1 January 2004 - 31 December 2006, altogether eighty-seven patients were enrolled. Forty-one patients were randomly assigned to the XELIRI and fortysix patients to the FOLFIRI arm. There were no statistically significant differences in baseline characteristics between the two arms (Table 1). Median duration of neoadjuvant treatment was 5.0 (range 1.1 - 9.6) months in the XELIRI arm and 5.1 (range $0.1-9.7$ ) in the FOLFIRI arm ( $\mathrm{p}=$ 0.45 ). The median follow up was 17 months (range 139). The efficacy of both treatment arms (ORR, R0 resection rate) is shown in Table 2. At the time of evaluation by CT scans after the conclusion of neoadjuvant chemotherapy, the disease was expected to be resectable in $29 \%$ of patients in the XELIRI arm and in $44 \%$ of patients in the FOLFIRI arm $(\mathrm{p}=0.16)$. All of these patients underwent surgery. The R0 resection has been performed in $10(24 \%)$ of all patients in the XELIRI arm and in $11(24 \%)$ of all patients in the FOLFIRI arm ( $\mathrm{p}=0.83)$. 
Table I: Baseline clinical characteristics of patients in the XELIRI and FOLFIRI arms, data are shown as $\mathbf{n}$ (\%) or as $\mathbf{n}$ (range)

\begin{tabular}{|c|c|c|c|}
\hline & $\begin{array}{c}\text { XELIRI } \\
n=4 I\end{array}$ & $\begin{array}{c}\text { FOLFIRI } \\
n=46\end{array}$ & $\mathbf{p}$ \\
\hline Median age-years & $63(47-75)$ & $62(34-75)$ & 0.33 \\
\hline \multicolumn{4}{|l|}{ Gender } \\
\hline Male & $26(63 \%)$ & 27 (59\%) & 0.38 \\
\hline Female & $15(37 \%)$ & $19(41 \%)$ & 0.40 \\
\hline \multicolumn{4}{|l|}{ WHO performance status } \\
\hline Performance status 0 & 31 (75\%) & $36(78 \%)$ & 0.82 \\
\hline Performance status I & $10(25 \%)$ & $10(22 \%)$ & 0.85 \\
\hline \multicolumn{4}{|l|}{ Primary tumour } \\
\hline Colon & $30(73 \%)$ & $40(87 \%)$ & 0.23 \\
\hline Rectum & II (27\%) & $6(13 \%)$ & 0.22 \\
\hline \multicolumn{4}{|l|}{ Initial stage of disease at diagnosis } \\
\hline Stage I & 0 & I (2\%) & - \\
\hline Stage 2 & $7(17 \%)$ & $3(7 \%)$ & 0.21 \\
\hline Stage 3 & $6(15 \%)$ & $14(30 \%)$ & 0,07 \\
\hline Stage 4 & $28(68 \%)$ & $28(61 \%)$ & 1.0 \\
\hline \multicolumn{4}{|l|}{ Previous adjuvant treatment* } \\
\hline Yes & $6(15 \%)$ & $10(22 \%)$ & 0.32 \\
\hline No & $35(85 \%)$ & $36(78 \%)$ & 0.91 \\
\hline \multicolumn{4}{|l|}{ Number of liver metastases } \\
\hline $1-4$ & $15(37 \%)$ & II (24\%) & 0.43 \\
\hline $5-10$ & $8(19 \%)$ & $10(22 \%)$ & 0.81 \\
\hline$>10$ & $18(44 \%)$ & $25(54 \%)$ & 0.28 \\
\hline \multicolumn{4}{|l|}{ Liver involvement (\%) } \\
\hline$<25$ & $14(34 \%)$ & $10(22 \%)$ & 0.41 \\
\hline $25-50$ & $13(32 \%)$ & $14(30 \%)$ & 0.85 \\
\hline$>50$ & $14(34 \%)$ & $21(45 \%)$ & 0.24 \\
\hline Median size of liver metastases (cm) & $4.0(1.5-12.4)$ & $5.0(0.5-15.0)$ & 0.10 \\
\hline Bilateral liver metastases, $\mathbf{n}(\%)$ & $35(85 \%)$ & $37(80 \%)$ & 0.81 \\
\hline \multicolumn{4}{|l|}{ Baseline CEA } \\
\hline Normal & $8(19 \%)$ & $10(22 \%)$ & 0.64 \\
\hline$>3.5 \mu \mathrm{g} / \mathrm{l}$ & $33(81 \%)$ & $36(78 \%)$ & 0.72 \\
\hline \multicolumn{4}{|l|}{ Baseline LDH } \\
\hline Normal & $25(61 \%)$ & 18 (39\%) & 0.28 \\
\hline$>4.12 \mu \mathrm{kat} / \mathrm{l}$ & $15(37 \%)$ & $26(56 \%)$ & 0.09 \\
\hline \multicolumn{4}{|l|}{ Reasons for initial unresectability } \\
\hline Location of metastases & $10(24 \%)$ & $8(17 \%)$ & 0.52 \\
\hline Number of metastases & $25(61 \%)$ & $30(65 \%)$ & 0.71 \\
\hline Size of metastases & $6(15 \%)$ & $8(17 \%)$ & 0.84 \\
\hline
\end{tabular}

* Adjuvant treatment with chemotherapy \pm radiotherapy

Table 2: Efficacy of treatment in the XELIRI and FOLFIRI arms (response rate, R0 resection* rate), data are shown as n (\%)

\begin{tabular}{|c|c|c|c|}
\hline & $\begin{array}{c}\text { XELIRI } \\
n=4 I\end{array}$ & $\begin{array}{c}\text { FOLFIRI } \\
n=46\end{array}$ & $\mathbf{p}$ \\
\hline Complete response & $5(12 \%)$ & I (2\%) & 0.10 \\
\hline Partial response & $15(37 \%)$ & $21(46 \%)$ & 0.32 \\
\hline Objective response & $20(49 \%)$ & $22(48 \%)$ & 0.76 \\
\hline Stagnation & $12(29 \%)$ & $10(22 \%)$ & 0.67 \\
\hline Progressive disease & $7(17 \%)$ & II (24\%) & 0.35 \\
\hline R0 resection* & $10(24 \%)$ & II $(24 \%)$ & 0.83 \\
\hline Complete response $+\mathrm{R} 0$ resection & $15(37 \%)$ & $12(26 \%)$ & 0.56 \\
\hline
\end{tabular}

R0 resection* - > $10 \mathrm{~mm}$ tumour-free resection margin on histology 
The PFS of patients in the XELIRI and FOLFIRI arms is shown in Figure 1. The median PFS for the patients in the XELIRI arm was 10.3 months (95\% CI: 9.1-11.5) and 9.3 months (95\% CI: 6.7-12.0) in the FOLFIRI arm ( $\mathrm{p}=$ 0.78 ). The OS of patients in the XELIRI and FOLFIRI arms is shown in Figure 2. The median OS for the XELIRI arm was 30.7 months (95\% CI: 19.5-41.9) and for the FOLFIRI arm 16.6 months (95\% CI: 7.9-25.3) $(\mathrm{p}=0.16)$.

At the time of the analysis, of 21 patients who underwent R0 resection of liver metastases, $19(90 \%)$ were alive: in the XELIRI arm, 9 out of $10(90 \%)$ patients, and in the FOLFIRI arm, 10 out of $11(91 \%)(\mathrm{p}=0.82)$. At the time of the analysis, CR after chemotherapy was evident in 4 out of $6(67 \%)$ patients: in the XELIRI arm, 3 out of 5 $(60 \%)$ patients, and in the FOLFIRI arm, $1 / 1$ patient $(\mathrm{p}=$ $0.32)$.
Adverse events of any grade in the treatment the XELIRI and FOLFIRI arms are shown in Table 3. The majority of adverse events were of grade I or II. The adverse events of grade 3 or 4 in the XELIRI arm were diarrhoea (7\%), neutropenia $(5 \%)$, and acute coronary syndrome (2\%). In the FOLFIRI arm, the adverse events of grade 3 or 4 were neutropenia $(13 \%)$, diarrhoea $(6 \%)$, acute coronary syndrome $(4 \%)$, and ischemic stroke $(2 \%)$. No hand-foot syndrome of grade 3 occurred in any of the two arms. Dose reduction due to adverse events was required in $12 \%$ of patients in the XELIRI arm and in $22 \%$ of patients in the FOLFIRI arm $(p=0.20)$. The therapy was completed as planned in $90 \%$ of patients in the XELIRI arm and in $87 \%$ of patients in the FOLFIRI arm $(\mathrm{p}=0.85)$. The diagnosis of steatohepatitis from histology reports at liver surgery was present in $6 / 12(50 \%)$ of patients in the XELIRI arm and in $8 / 20(40 \%)$ of patients in the FOLFIRI arm ( $\mathrm{p}=$

\section{Progression free survival (PFS)}

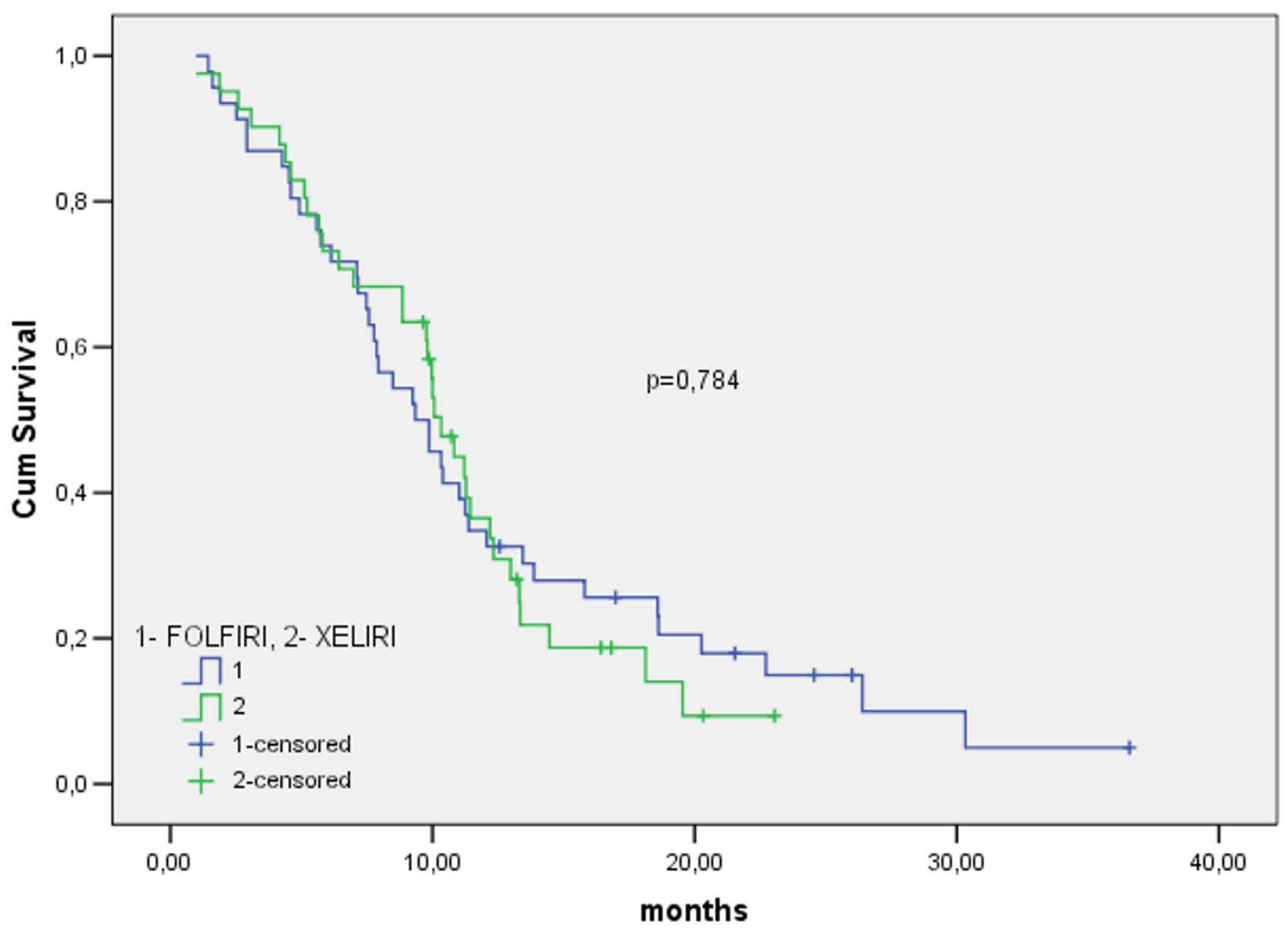

\section{Figure I}

The PFS of patients in the XELIRI and FOLFIRI arms (months). 


\section{Overall survival (OS)}

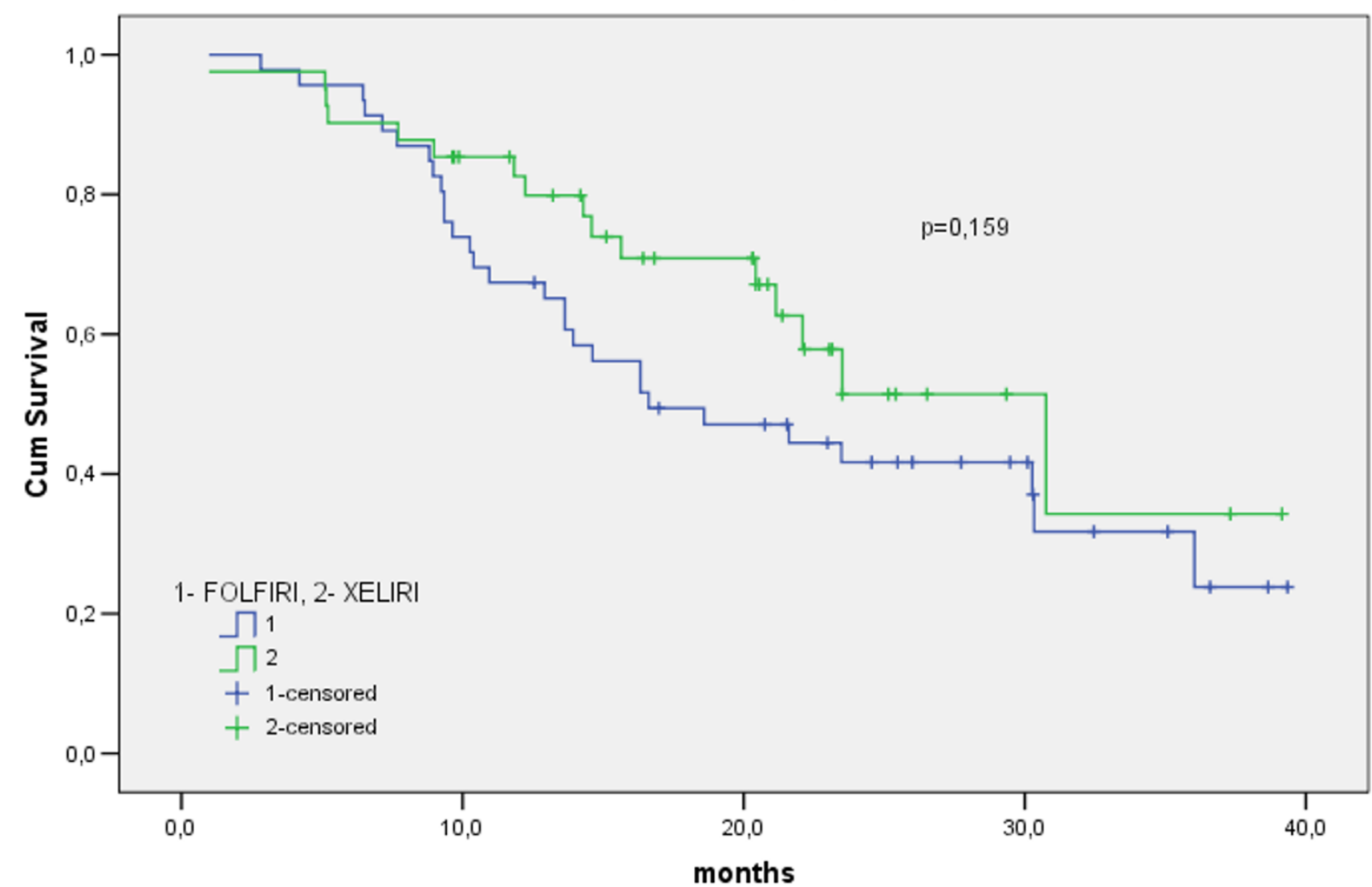

Figure 2

The OS of patients in the XELIRI and FOLFIRI arms (months).

0.59). Steatohepatitis was present in median of $65 \%$ (range 20\% - 90\%) of liver tissue in the XELIRI arm and in median of $55 \%$ (range $10 \%-70 \%$ ) in the FOLFIRI arm $(\mathrm{p}=0.59)$. The intensity of steatohepatitis was either mild ( 3 patients) or modest ( 3 patients) in the XELIRI arm, and mild (5 patients), modest ( 1 patient) and severe (2 patients $)$ in the FOLFIRI arm $(\mathrm{p}=0.30)$. None of patients with steatohepatitis had any additional perioperative comorbidity after liver surgery. There were no treatmentrelated deaths in any of the two arms of patients.

At relapse, all patients received at least one line of chemotherapy: oxaliplatin-based second-line therapy received $85 \%$ of patients in the XELIRI arm and $62 \%$ of patients in the FOLFIRI arm ( $\mathrm{p}=0.59)$, cetuximab-based second-line therapy received $5 \%$ of patients in the XELIRI arm and $12 \%$ of patients in the FOLFIRI arm $(\mathrm{p}=0.32)$.

\section{Discussion}

The present study reports the comparison of efficacy, safety and survival between the XELIRI and FOLFIRI regimen in the neoadjuvant treatment of patients with MCRC.

The current single-institution randomised phase II trial demonstrated that the XELIRI regimen is effective in the neoadjuvant treatment of patients with MCRC and that it has acceptable toxicity, when compared to the FOLFIRI regimen. Both primary end-points were practically identical between the two arms. The relatively high rate of objective response (49\% and $48 \%$ ) in both arms is in the range of expected, since all patients had liver-only metastases. The rate of $\mathrm{R} 0$ resection (24\%) of liver metastases in our study is one of the highest reported. We believe that both chemotherapy regimens are effective since, at the end of treatment, $37 \%$ of patients were without any evidence of the disease (either complete remission achieved with chemotherapy or R0 resection of liver metastases) in the XELIRI regimen and $26 \%$ of patients in the FOLFIRI regimen. 
Table 3: Adverse events regarding CTCAE-3 in the XELIRI and FOLFIRI arms, data are shown as $n$ (\%)

\begin{tabular}{|c|c|c|c|}
\hline & $\begin{array}{l}\text { XELIRI } \\
N=4 I\end{array}$ & $\begin{array}{c}\text { FOLFIRI } \\
n=46\end{array}$ & $\mathbf{p}$ \\
\hline \multicolumn{4}{|l|}{ Hand-foot syndrome } \\
\hline Grade I & $4(10 \%)$ & 0 & 0.18 \\
\hline Grade 2 & $2(5 \%)$ & 0 & 0.10 \\
\hline Grade 3 & 0 & 0 & - \\
\hline \multicolumn{4}{|l|}{ Diarrhoea } \\
\hline Grade I & I (2\%) & $4(8 \%)$ & 0.18 \\
\hline Grade 2 & $4(10 \%)$ & 7 (I5\%) & 0.37 \\
\hline Grade 3 & I (2\%) & $2(4 \%)$ & 0.56 \\
\hline Grade 4 & $2(5 \%)$ & I (2\%) & 0.56 \\
\hline \multicolumn{4}{|l|}{ Nausea } \\
\hline Grade I & $3(7 \%)$ & $8(17 \%)$ & 0.13 \\
\hline Grade 2 & $6(15 \%)$ & I (2\%) & 0.06 \\
\hline Grade 3 & 0 & 0 & - \\
\hline Grade 4 & 0 & 0 & - \\
\hline \multicolumn{4}{|l|}{ Fatigue } \\
\hline Grade I & $3(7 \%)$ & $4(9 \%)$ & 0.70 \\
\hline Grade 2 & I (2\%) & $2(4 \%)$ & 0.56 \\
\hline Grade 3 & 0 & 0 & - \\
\hline Grade 4 & 0 & 0 & - \\
\hline \multicolumn{4}{|l|}{ Neutropenia } \\
\hline Grade I & $3(7 \%)$ & $2(4 \%)$ & 0.65 \\
\hline Grade 2 & $2(5 \%)$ & $12(26 \%)$ & 0.008 \\
\hline Grade 3 & I (2\%) & 5 (II\%) & 0.10 \\
\hline Grade 4 & I (2\%) & I (2\%) & 1.0 \\
\hline Thrombopenia of any grade & 0 & 0 & - \\
\hline Ischemic stroke & 0 & I (2\%) & - \\
\hline Acute coronary syndrome & I (2\%) & $2(4 \%)$ & 0.56 \\
\hline Death* & 0 & 0 & - \\
\hline
\end{tabular}

* Patients who died within 30 days after the last cycle of chemotherapy or liver surgery

The PFS of 9-10 months in the patients in our study was similar to the PFS of patients with liver only metastases treated with the FOLFIRI regimen in a recently reported large randomised the CRYSTAL trial [17]. In the CRYSTAL trial, however, the secondary resection rate of liver metastases in 134 patients with liver-only metastases was only $4.5 \%$. Since the majority of patients in the CRYSTAL trial had extra-hepatic disease, it is possible that the low rate of secondary resection of liver-only metastases was due to the lack of active searching for resectable liver metastases after neoadjuvant chemotherapy by the multidisciplinary team consisting of a liver surgeon, radiologist and medical oncologist. We believe that this multidisciplinary approach is essential for an optimal treatment outcome of patients with liver-only metastases of MCRC.

Despite the high R0 resection rate in both our study arms and high survival of patients following R0 resection in our study, the median OS of all patients in the FOLFIRI arm is one of the lowest ever reported for this regimen (16.6 months). It is possible that poor median OS in our FOLFIRI arm is due to the fact that only $62 \%$ of patients in this arm received an oxaliplatin-based second line regimen at progression of the disease. For the patients with MCRC who receive all three chemotherapeutic drugs (irinotecan, oxaliplatin, and fluoropyrimidine), the expected median OS is $20-22$ months $[18,19]$.

It is noteworthy that the XELIRI regimen showed acceptable toxicity. To our surprise, no grade 3 hand-foot syndrome, a well-known adverse event of capecitabine, occurred in the XELIRI arm. This is possibly due to the lower daily dose of capecitabine in the XELIRI regimen than in the capecitabine monotherapy. During the treatment with irinotecan and capecitabine, diarrhoea is a frequent adverse event when these drugs are used as single agents. In our study, the rate of grade 3 or 4 diarrhoea in the XELIRI arm was also low. This is in concordance with the European CAIRO trial [20], which showed no additional grade 3 or 4 diarrhoea in the patients in the XELIRI arm as compared to the sequential therapy with capecitabine and irinotecan monotherapy. In contrast, in the recently published American trial BICC-C [21], the incidence of grade 3 or 4 diarrhoea was present in $48 \%$ of patients in the XELIRI arm. The reason for these differences in diarrhoea incidences among these trials is not known.

The neoadjuvant chemotherapy is known to cause liver toxicity. For the irinotecan-based regimens, the usual pathological abnormality of liver cells is steatohepatitis. Because of liver toxicity, the resection of liver metastases should be performed as soon as the metastases become resectable with neoadjuvant chemotherapy. There were signs of steatohepatitis in both arms of patients, but no additional co-morbidities were observed after liver resections in the patients of either arm.

In the recent years, three targeted drugs became available for the treatment of patients with MCRC (bevacizumab, cetuximab, panitumumab). Since best clinical benefit of these drugs is achieved in combination with chemotherapy, they are usually used with different chemotherapy regimens. The combination of targeted therapy with different chemotherapy regimens showed favourable objective response rates when compared to chemotherapy alone in phase II/III studies $[17,22]$. In the neoadjuvant setting, the regimens with higher objective response rates are preferred as it is believed that higher objective response rates lead to higher R0 resection rates of liver metastases. Therefore, the combination of targeted agents with chemotherapy is generally applied in the neoadjuvant setting. Because of this, our study was prematurely closed soon after bevacizumab had become available in our country. We believe that the XELIRI regimen should be considered to be part of phase II trials where the targeted drugs, like cetuximab or bevacizumab, are used in 
combination with chemotherapy. Outside clinical trials, in small subset of patients, where the targeted agents are contraindicated or not available to the patients, the XELIRI regimen could be used as an alternative to the FOLFIRI regimen in the neoadjuvant setting of patients with liver-only metastases of MCRC.

\section{Conclusion}

Our study showed that the XELIRI regimen is effective with acceptable toxicity when compared to the FOLFIRI regimen in neoadjuvant treatment of patients with unresectable liver-only metastases of MCRC. XELIRI regimen merits further evaluation in phase II trials in combination with targeted drugs in patients with MCRC.

\section{Competing interests}

The authors declare that they have no competing interests.

\section{Authors' contributions}

ES made a substantial contribution to the conception, design, and coordination of the study, to the treatment of patients, interpretation of data; he performed statistical analysis, and was involved in drafting the manuscript. MR made a substantial contribution to the treatment of patients and helped to draft the manuscript. $\mathrm{ZH}$ made a substantial contribution to the treatment of patients and helped to draft the manuscript. JO made a substantial contribution to the conception, design and coordination of the study and to the treatment of patients, and helped to draft the manuscript. All authors read and approved the final manuscript.

\section{References}

I. Abdalla EK, Vauthey JN, Ellis LM, Ellis V, Pollock R, Broglio KR, Hess $K$, Curley SA: Recurrence and outcomes following hepatic resection, radiofrequency ablation, and combined resection/ ablation for colorectal liver metastases. Ann Surg 2004, 239:818-825.

2. Wei AC, Greig PD, Grant D, Taylor B, Langer B, Gallinger S: Survival after hepatic resection for colorectal metastases: a 10 year experience. Ann Surg Oncol 2006, 13:668-676.

3. Simmonds PC, Primrose JN, Colquitt JL, Garden OJ, Poston GJ, Rees $M$ : Surgical resection of hepatic metastases from colorectal cancer: a systematic review of published studies. $\mathrm{Br} J$ Cancer 2006, 94:982-999.

4. Cummings LC, Payes JD, Cooper GS: Survival after hepatic resection in metastatic colorectal cancer: a population-based study. Cancer 2007, 109:718-726.

5. Van Cutsem E, Nordlinger B, Adam R, Köhne CH, Pozzo C, Poston G, Ychou M, Rougier P: European Colorectal Metastases Treatment. Towards a pan-European consensus on the treatment of patients with colorectal liver metastases. Eur J Cancer 2006, 42:22I2-2221.

6. Masi G, Cupini S, Marcucci L, Cerri E, Loupakis F, Allegrini G, Brunetti IM, Pfanner E, Viti M, Goletti O, Filipponi F, Falcone : Treatment with 5-fluorouracil/folinic acid, oxaliplatin, and irinotecan enables surgical resection of metastases in patients with initially unresectable metastatic colorectal cancer. Ann Surg Oncol 2006, 13:58-56.

7. Alberts SR, Horvath WL, Sternfeld WC, Goldberg RM, Mahoney MR, Dakhil SR, Levitt R, Rowland K, Nair S, Sargent DJ, Donohue : Oxaliplatin, Fluorouracil, and Leucovorin for Patients With Unresectable Liver-Only Metastases From Colorectal Cancer: A
North Central Cancer Treatment Arm Phase II Study. J Clin Oncol 2005, 23:9243-9249.

8. Barone C, Nuzzo G, Cassano A, Basso M, Schinzari G, Giuliante F, D'Argento E, Trigila N, Astone A, Pozzo : Final analysis of colorectal cancer patients treated with irinotecan and 5-fluorouracil plus folinic acid neoadjuvant chemotherapy for unresectable liver metastases. Br J Cancer 2007, 97: I035-I039.

9. Porschen R, Arkenau HT, Kubicka S, Greil R, Seufferlein T, Freier W, Kretzschmar A, Graeven U, Grothey A, Hinke A, Schmiegel W, Schmoll HJ, AIO Colorectal Study Arm: Capecitabine Plus Oxaliplatin Compared With Fluorouracil and Leucovorin Plus Oxaliplatin: A Randomized Comparison in Metastatic Colorectal Cancer - A Final Report of the AIO Colorectal Study Arm. J Clin Oncol 2007, 25:4217-4223.

10. Diaz-Rubio E, Tabernero J, Gomez-Espana A, Massutí B, Sastre J, Chaves M, Abad A, Carrato A, Queralt B, Reina JJ, Maurel J, GonzálezFlores E, Aparicio J, Rivera F, Losa F, Aranda E, Spanish Cooperative Arm for the Treatment of Digestive Tumors Trial: Phase III Study of Capecitabine Plus Oxaliplatin Versus Continuous-Infusion Fluorouracil Plus Oxaliplatin As First-Line Therapy in Metastatic Colorectal Cancer: Final Report of the Spanish Cooperative Arm for the Treatment of Digestive Tumors Trial. J Clin Oncol 2007, 25:4224-4230.

II. Patt YZ, Lee FC, Leibmann JE, Diamandidis D, Eckhardt SG, Javle M, Justice GR, Keiser W, Salvatore JR, Bexon A, Lin E: Capecitabine plus 3-weekly irinotecan (XELIRI regimen) as first-line chemotherapy for metastatic colorectal cancer. Am J Clin Oncol 2007, 30:350-357.

12. Bajetta E, Di Bartolomeo M, Mariani L, Cassata A, Artale S, Frustaci S, Pinotti G, Bonetti A, Carreca I, Biasco G, Bonaglia L, Marini G, lannelli A, Cortinovis D, Ferrario E, Beretta E, Lambiase A, Buzzoni R, Italian Trials in Medical Oncology (I.T.M.O.) Arm: Randomized multicenter Phase II trial of two different schedules of irinotecan combined with capecitabine as first-line treatment in metastatic colorectal carcinoma. Cancer 2004, 100:279-287.

13. Borner MM, Bernhard J, Dietrich D, Popescu R, Wernli M, Saletti P, Rauch D, Herrmann R, Koeberle D, Honegger H, Brauchli P, Lanz D, Roth AD, Swiss Arm for Clinical Cancer Research (SAKK), Berne, Switzerland: A randomized phase II trial of capecitabine and two different schedules of irinotecan in first-line treatment of metastatic colorectal cancer: efficacy, quality-of-life and toxicity. Ann Oncol 2005, 16:282-288.

14. Rea DW, Nortier JW, Ten Bokkel Huinink WW, Falk S, Richel DJ, Maughan T, Groenewegen G, Smit JM, Steven N, Bakker JM, Semiond $D$, Kerr DJ, Punt Cl: A phase I/II and pharmacokinetic study of irinotecan in combination with capecitabine as first-line therapy for advanced colorectal cancer. Ann Oncol 2005, 16:1123-1132.

15. Therasse P, Arbuck SG, Eisenhauer EA, Wanders J, Kaplan RS, Rubinstein L, Verweij J, Van Glabbeke M, van Oosterom AT, Christian MC, Gwyther SG: New guidelines to evaluate the response to treatment in solid tumors. European Organization for Research and Treatment of Cancer, National Cancer Institute of the United States, National Cancer Institute of Canada. J Natl Cancer Inst 2000, 92:205-216.

16. Kaplan E, Meier P: Nonparametric estimation from incomplete observations. J Am Stat Assoc 1958, 53:457-48I.

17. Van Cutsem E, Bodoky G, Roh JK, Folprecht G, Park YS, Van Laethem JL, Raoul JL, Ciardiello F, Lebrun P, Rougier P: CRYSTAL, a randomized phase III trial of cetuximab plus FOLFIRI vs. FOLFIRI in first-line metastatic colorectal cancer (mCRC) [abstract]. Eur J Cancer 2007, 5(Suppl 4):235.

18. Tournigand C, Andre T, Achille E, Lledo G, Flesh M, Mery-Mignard D, Quinaux E, Couteau C, Buyse M, Ganem G, Landi B, Colin P, Louvet $C$, de Gramont A: FOLFIRI followed by FOLFOX6 or the reverse sequence in advanced colorectal cancer: a randomized GERCOR study. / Clin Oncol 2004, 22:229-237.

19. Colucci G, Gebbia V, Paoletti G, Giuliani F, Caruso M, Gebbia N, Carteni G, Agostara B, Pezzella G, Manzione L, Borsellino N, Misino A, Romito S, Durini E, Cordio S, Di Seri M, Lopez M, Maiello E, Montemurro S, Cramarossa A, Lorusso V, Di Bisceglie M, Chiarenza M, Valerio MR, Guida T, Leonardi V, Pisconti S, Rosati G, Carrozza F, Nettis G, Valdesi M, Filippelli G, Fortunato S, Mancarella S, Brunetti $C$ : Phase III randomized trial of FOLFIRI versus FOLFOX4 in the treatment of advanced colorectal cancer: a multicenter 
study of the Gruppo Oncologico Dell'Italia Meridionale. J Clin Oncol 2005, 23:4866-75.

20. Koopman M, Antonini NF, Douma J, Wals J, Honkoop AH, Erdkamp FL, de Jong RS, Rodenburg C], Vreugdenhil G, Loosveld OJ, van Bochove A, Sinnige HA, Creemers GJ, Tesselaar ME, Slee PH, Werter MJ, Mol L, Dalesio O, Punt CJ: Sequential versus combination chemotherapy with capecitabine, irinotecan, and oxaliplatin in advanced colorectal cancer (CAIRO): a phase III randomised controlled trial. Lancet 2007, 370: I35-142.

21. Fuchs CS, Marshall J, Mitchell E, Wierzbicki R, Ganju V, Jeffery M, Schulz J, Richards D, Soufi-Mahjoubi R, Wang B, Barrueco J: Randomized, controlled trial of irinotecan plus infusional, bolus, or oral fluoropyrimidines in first-line treatment of metastatic colorectal cancer: results from the BICC-C Study. J Clin Oncol 2007, 25:4779-4786.

22. Folprecht G, Gruenberger T, Hartmann JT, Lordick F, Stoehlmacher J, Bechstein W, Ockert D, Hermann T, Liersch T, Koehne C: Randomized multicenter study of cetuximab plus FOLFOX of plus FOLFIRI in neoadjuvant treatment of non-resectable colorectal liver metastases (CELIM-study). Ann Oncol 2007, 19(Suppl 8):viii 168.

\section{Pre-publication history}

The pre-publication history for this paper can be accessed here:

http://www.biomedcentral.com/1471-2407/9/120/pre pub

Publish with Biomed Central and every scientist can read your work free of charge

"BioMed Central will be the most significant development for disseminating the results of biomedical research in our lifetime. "

Sir Paul Nurse, Cancer Research UK

Your research papers will be:

- available free of charge to the entire biomedical community

- peer reviewed and published immediately upon acceptance

- cited in PubMed and archived on PubMed Central

- yours - you keep the copyright 